\title{
Successful Bone Union Following Calcium Phosphate Cement-Assisted Percutaneous Transpedicular Balloon Kyphoplasty of a Large Interbody Cleft on Long-term Hemodialysis Patient
}

\author{
Shigeo Ishiguro', Masaya Tsujii², Akihiro Sudo² \\ 'Department of Orthopaedic Surgery, Oyamada Memorial Spa Hospital, Yokkaichi, Japan \\ ${ }^{2}$ Department of Orthopaedic Surgery, Mie University Graduate School of Medicine, Mie, Japan
}

A 68-year-old diabetic man, who had been on dialysis for 3 years, suffered a five week history of severe back pain that was unresponsive to bed rest, analgesics, and bracing. The vertebral cleft formed by an injury gradually increased in size on sequential plain films. Hence, he underwent calcium phosphate cement-assisted percutaneous transpedicular balloon kyphoplasty to treat a painful interbody vacuum cleft. Immediate pain relief and firm bone union were obtained.

Key Words: Hemodialysis, Adjacent vertebral fractures, Large vertebral cleft, Calcium phosphate cement

\section{Introduction}

A patient on long-term hemodialysis underwent calcium phosphate cement (CPC)-assisted percutaneous transpedicular balloon kyphoplasty to treat a painful interbody vacuum cleft. Immediate pain relief and firm bone union were obtained.

Although acute lethal complications in vertebroplasty or kyphoplasty using polymethylmethacrylate (PMMA) - such as in pulmonary embolism due to elevated pressure during PMMA injection [1] or catastrophic cement extravasations [2] - can be prevented with adequate safeguards, in 10 to 20 percent of cases adjacent vertebral fractures occur and are unpreventable [3]. Moreover, refracture with cement extrusion after PMMA vertebroplasty via a unipedicular approach has been recently reported in a hemodialysis patient a cleft [4]. Here we describe a case of immediate pain relief and successful bone union following CPC-assist- ed percutaneous transpedicular balloon kyphoplasty for vertebral compression fracture with a large vertebral cleft in a patient with end-stage renal disease.

\section{Case Report}

A 68-year-old diabetic man, who had been on dialysis for 3 years, presented with a five week history of severe back pain that was unresponsive to bed rest, analgesics, and bracing. Lateral extension and lateral flexion radiographs revealed a T12 vertebral body fracture with an interbody vacuum cleft showing mobility. The cleft had been present right after an injury - falling on his buttocks - and gradually increased in size on sequential plain films (Fig. 1).

The patient was placed in the prone position under general anesthesia. Under C-arm confirmation, through a very small longitudinal skin stab incision on the bilateral pedicle, using a cannulated drilling system and a dilation technique, $1 \mathrm{~mL}$ syringes (JMS Co., Ltd., Tokyo, Japan) were set into

Received May 3, 2010; 1st Revised May 21, 2010; 2nd Revised Jun 4, 2010; Accepted Jun 8, 2010

Corresponding author: Shigeo Ishiguro, MD

Department of Orthopaedic Surgery, Oyamada Memorial Spa Hospital,

Yamada-cho, Yokkaichi city, Mie Prefecture, Japan

Tel: +81-59-328-1260, Fax:+81-59-328-3040, E-mail: stoneblack1496@syutaikai.jp 

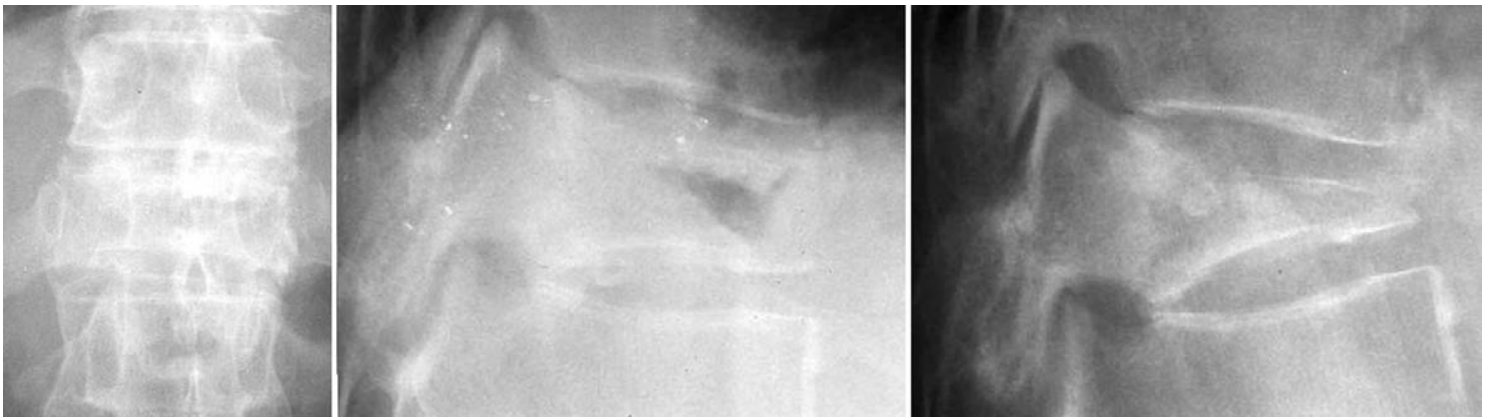

Fig. 1. Thoracic vertebral fracture shows mobility with progressively enlarging vacuum cleft.

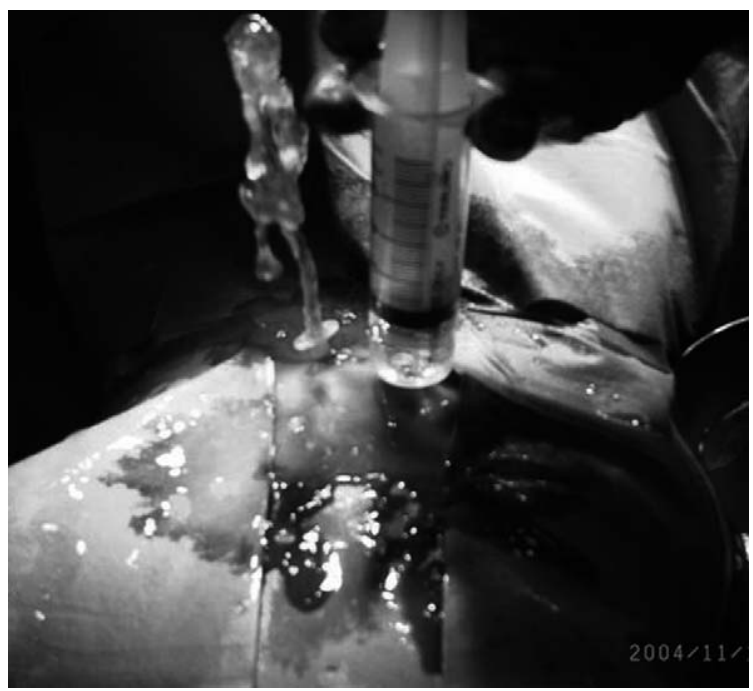

Fig. 2. Saline poured from one entrance pedicle flows out another exit.

both sides of the pedicle. The vertebral body was irrigated by saline, which was injected into one of the pedicles. A return stream was allowed to exit on the contralateral pedicle (Fig. 2). It was also irrigated from the opposite side of the pedicle. We routinely try to remove sequestered hematomas by saline irrigation so as to increase the potential space for CPC and prevent increased intra-vertebral body pressure, which can potentially cause pulmonary embolism and extravasation of CPC. Additionally, the presence of a hematoma and a low powder/liquid ratio in the CPC decreases the final compressive strength of the CPC [5]. These conditions may cause failure of CPC-assisted vertebroplasty.

A pediatric uromatic balloon, size 8 Fr (Medicon Co., Ltd., Osaka, Japan) was then introduced into the cavity and inflated using contrast medium (Fig. 3). Approximately $3 \mathrm{ml}$ of the contrast medium was necessary. The balloon inflation further enlarged the pre-existing fracture cavity and made it more apparent. Obstructing one side of the pedicle with a finger made it impossible to inject saline from the other side, and the cavity was considered to be closed except for the bilateral pedicle route.

Before starting to knead the $\mathrm{CPC}$, the pediatric uromatic balloon was once again introduced into the created cavity and inflated to achieve hemostasis inside the vertebral body. CPC (Biopex R, Mitsubishi Materials Co., Ltd., Tokyo, Japan) injections were done using a small hand-piece cement-gun after 1-3 minutes of kneading at room temperature. We injected $6 \mathrm{ml}$ of CPC and the surplus CPC flowed out of both syringes. The patient was able to begin ambulation on day four postoperatively using a brace. Fig. 4 shows a postoperative radiograph and a computed tomography (CT) scan. Postoperative correction loss continued until 6 weeks postoperatively. Bone union was obtained at 8 weeks postoperatively. Fig. 5 shows a 1 year postoperative radiograph and CT scan.

\section{Discussion}

Although most authors believe that a patient with a vertebral cleft is suitable for vertebroplasty [6], Wagner and Baskurt [4] reported that treatment for a vacuum cleft in an end-stage hemodialysis patient had an unfavorable clinical course. They found that, when filling a vertebral cleft in osteoporotic patients, it seems reasonable to assume that there is an additional risk of refracture of that level, because of the strength of the cement compared with the weakness of the surrounding bone.

Although the compressive strength of CPC (80 MPa) is lower than that of PMMA (99 MPa) [5,7,8], we estimated that CPC is more suitable than PMMA because an osteoporotic vertebral body has a lower compressive force than CPC. In a previous in vivo study, CPC was shown to be biocompatible in biological and biomechanical aspects with bone and soft tissue $[5,7,8]$. 

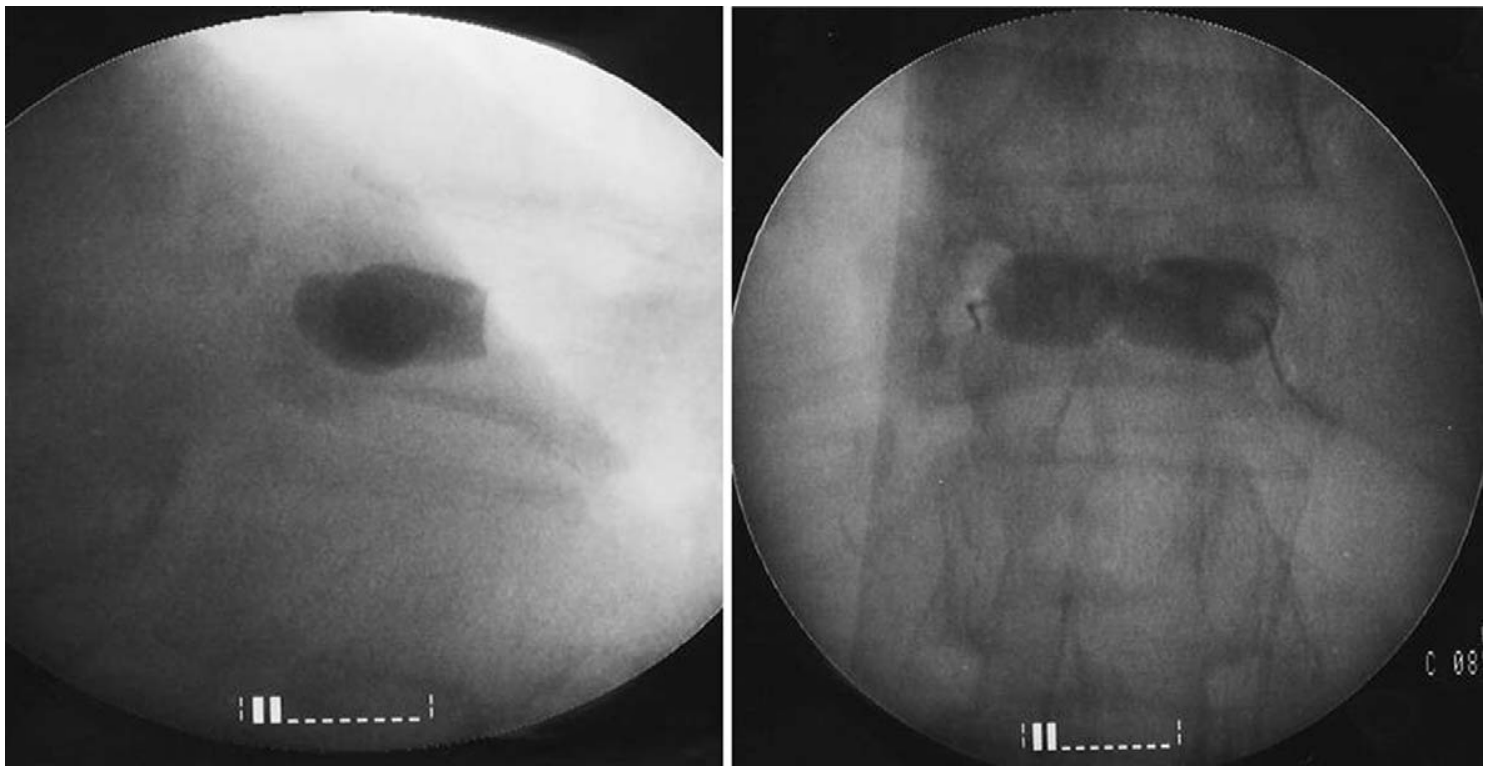

Fig. 3. Radiological image showing pediatric uromatic balloon, size 8 Fr (Medicon Co., Ltd., Osaka, Japan); preinflation diameter, $2.7 \mathrm{~mm}$; length, $30 \mathrm{~cm}$. Balloon was introduced into vacuum cleft and inflated by contrast medium. About $3 \mathrm{ml}$ of contrast medium was required to fully inflate each balloon to enlarge preexisting cavity.
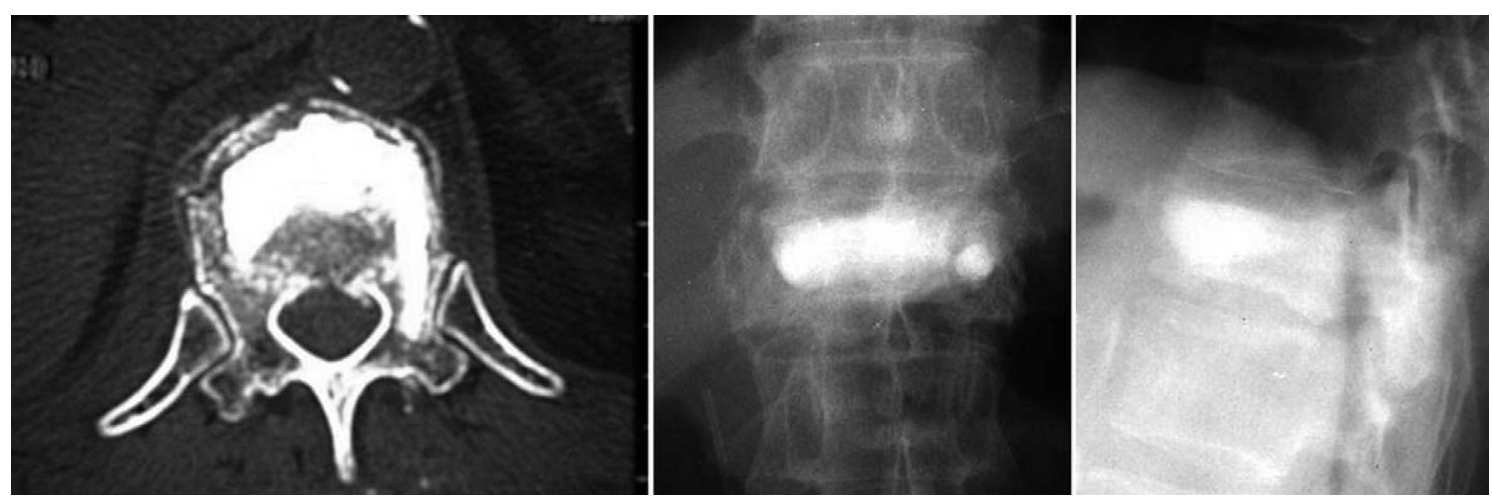

Fig. 4. Postoperative X-ray shows good reduction and postoperative computed tomography depicts linear calcium phosphate cement leakage around vertebral body.

In unipedicular ertebroplasy, the PMMA filling was terminated after a pressure end point was reached. This procedure seems safe and adequate in terms of preventing PMMA extravasation and pulmonary embolism. However, it is easy to imagine that a hematoma formation can result in elevated pressure and inadequate PMMA filling following cement fragmentation, scar formation, late collapse of PMMA and surrounding bone fracture.

Our bipedicular technique using the pediatric balloon, on the other hand, does not involve pressure elevation. Excessive local pressure during injection leads to cement extrusion throughout the defect of the posterior wall of the vertebral body into the spinal canal. Spacing of the vertebral body bilaterally through the bilateral pedicles decreases the risk of excessive local pressure, which might lead to cement extrusion and embolism.

Furthermore, a hematoma was spontaneously removed with CPC as surplus CPC stained with blood came out of the syringe in the opposite pedicle. This theory has already proven useful in other orthopaedic fields for fractures in hemodialysis patients and senile female patients $[9,10]$.

We have a valid reason why we did not use a commercial surgical kit including the ballooning tamp used in kyphoplasty. As the Japanese government has not approved it yet, Japanese clinical orthopaedic surgeons can not use it. To mimic the ballooning tamp, we used a pediatric uromatic balloon instead of the kyphoplasty kit. The pediatric uromatic balloon is made for continuous urethral catheteriza- 

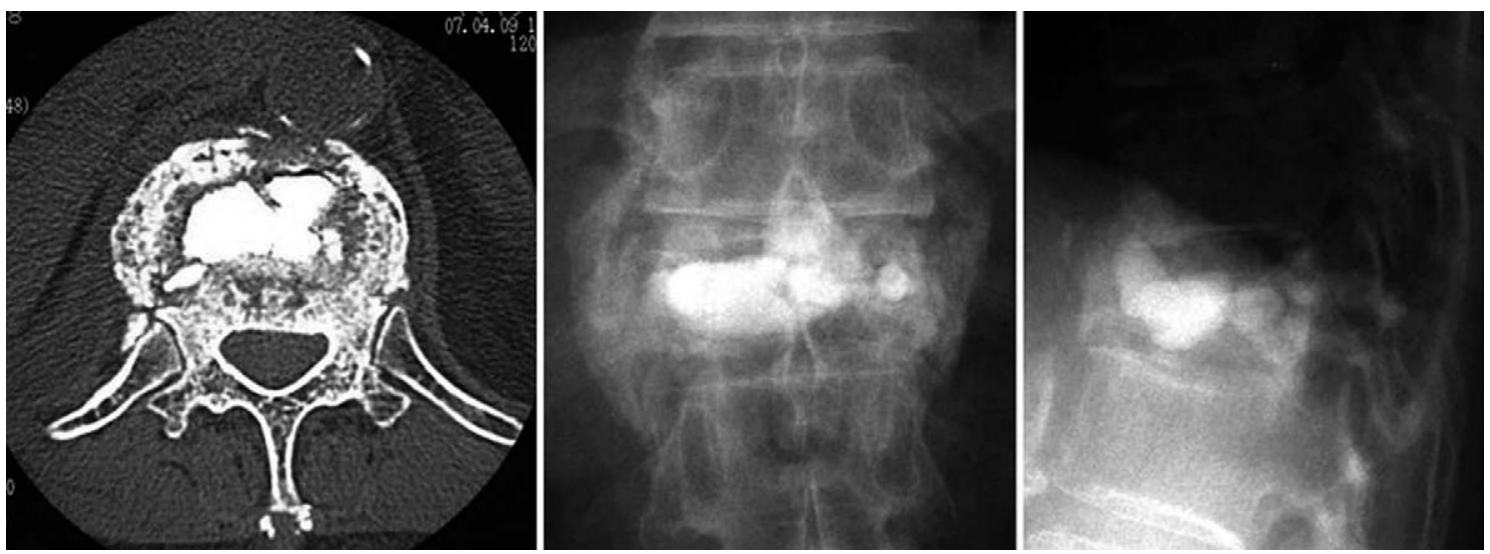

Fig. 5. One-year postoperatively, antero-posterior radiograph shows bone formation in vertebral body and bilateral osseous bridging around vertebral body, although lateral radiograph shows obvious postoperative correction loss. Computed tomography obtained at the time shows hyperosteogenesis around vertebral body conducted by calcium phosphate cement leakage.

tion for children and not tolerant enough to reduce the fracture. However, it has enough strength to enlarge the fracture cavity in the osteoporotic bone. And a possible advantage over the kyphoplasty kit may be its low price.

The postoperatively reduced vertebral body was collapsed again, even though some part of the CPC was converted into normal bone. We can not describe this as a favorable result. However, the hemodialysis patient was bed-ridden for one month period and he would not have recovered unless he had received our surgical rescue. Use of our noble bipedicular approach is clinically applicable even in an osteoporotic compression fracture in a hemodialysis patient because it is less invasive, and more cost-effective and safe.

\section{REFERENCES}

1. Tozzi P, Abdelmoumene Y, Corno AF, Gersbach PA, Hoogewoud HM, von Segesser LK. Management of pulmonary embolism during acrylic vertebroplasty. Ann Thorac Surg 2002;74:1706-8.

2. Hardouin P, Grados F, Cotten A, Cortet B. Should percutaneous vertebroplasty be used to treat osteoporotic fractures? An update. Joint Bone Spine 2001;68:216-21.

3. Lavelle WF, Cheney R. Recurrent fracture after vertebral kyphoplasty. Spine J 2006;6:488-93.

4. Wagner AL, Baskurt E. Refracture with cement extrusion following percutaneous vertebroplasty of a large interbody cleft. AJNR Am J Neuroradiol 2006;27:230-1.

5. Kurashina K, Kurita H, Hirano M, Kotani A, Klein CP, de Groot K. In vivo study of calcium phosphate cements: implantation of an alpha-tricalcium phosphate/dicalcium phosphate dibasic/tetracalcium phosphate monoxide cement paste. Biomaterials 1997;18:539-43.

6. Lane JI, Maus TP, Wald JT, Thielen KR, Bobra S, Luetmer $\mathrm{PH}$. Intravertebral clefts opacified during vertebroplasty: pathogenesis, technical implications, and prognostic significance. AJNR Am J Neuroradiol 2002;23:1642-6.

7. Kurashina K, Kurita H, Kotani A, Takeuchi H, Hirano M. In vivo study of a calcium phosphate cement consisting of alpha-tricalcium phosphate/dicalcium phosphate dibasic/ tetracalcium phosphate monoxide. Biomaterials 1997;18: 147-51.

8. Kindt-Larsen T, Smith DB, Jensen JS. Innovations in acrylic bone cement and application equipment. J Appl Biomater 1995;6:75-83.

9. Iida K, Sudo A, Ishiguro S. Clinical and radiological results of calcium phosphate cement-assisted balloon osteoplasty for Colles' fractures in osteoporotic senile female patients. J Orthop Sci 2010;15:204-9.

10. Ishiguro S, Oota Y, Sudo A, Uchida A. Calcium phosphate cement-assisted balloon osteoplasty for a Colles' fracture on arteriovenous fistula forearm of a maintenance hemodialysis patient. J Hand Surg Am 2007;32:821-6. 